\title{
THE LIMITING BEHAVIOUR OF HERMITIAN-YANG-MILLS FLOW OVER COMPACT NON-KÄHLER MANIFOLDS
}

\author{
YANCI NIE AND XI ZHANG
}

\begin{abstract}
In this paper, we analyze the asymptotic behaviour of the Hermitian-Yang-Mills flow over a compact non-Kähler manifold $(X, g)$ with the Hermitian metric $g$ satisfying the Gauduchon and Astheno-Kähler condition.
\end{abstract}

\section{INTRODUCTION}

Let $X$ be an $n$-dimensional compact complex manifold and $g$ a Hermitian metric with associated $(1,1)$-form $\omega . g$ is called to be Gauduchon if $\omega$ satisfies $\partial \bar{\partial} \omega^{n-1}=0$. It has been proved by Gauduchon ([12]) that if $X$ is compact, there exists a Gauduchon metric in the conformal class of every Hermitian metric $g$. If $\partial \bar{\partial} \omega^{n-2}=0$, the Hermitian metric $g$ is said to be Astheno-Kähler which was introduced by Jost and Yau in [15].

Let $(X, \omega)$ be an $n$-dimensional compact complex manifold with $\partial \bar{\partial} \omega^{n-1}=0$ and $(L, h)$ a Hermitian line bundle over $X$. The $\omega$-degree of $L$ is defined by

$$
\operatorname{deg}_{\omega}(L):=\int_{X} c_{1}\left(L, A_{h}\right) \wedge \frac{\omega^{n-1}}{(n-1) !},
$$

where $c_{1}\left(L, A_{h}\right)$ is the first Chern form of $L$ associated with the induced Chern connection $A_{h}$. Since $\partial \bar{\partial} \omega^{n-1}=0, \operatorname{deg}_{\omega}(L)$ is well defined and independent of the choice of metric $h([18$, p. 34$35])$. Now given a rank $s$ coherent analytic sheaf $\mathcal{F}$, we consider the determinant line bundle $\operatorname{det} \mathcal{F}=\left(\wedge^{s} \mathcal{F}\right)^{* *}$ associated with $\mathcal{F}$. Define the $\omega$-degree of $\mathcal{F}$ by

$$
\operatorname{deg}_{\omega}(\mathcal{F}):=\operatorname{deg}_{\omega}(\operatorname{det} \mathcal{F}) \text {. }
$$

If $\mathcal{F}$ is non-trivial and torsion free, the $\omega$-slope of $\mathcal{F}$ is defined by

$$
\mu_{\omega}(\mathcal{F})=\frac{\operatorname{deg}_{\omega}(\mathcal{F})}{\operatorname{rank}(\mathcal{F})}
$$

Let $\left(E, \bar{\partial}_{E}\right)$ be a rank $r$ holomorphic vector bundle over $(X, \omega)$. A Hermitian metric $H$ on $E$ is said to be $\omega$-Hermitian-Einstein if the Chern curvature $F_{H}$ satisfies the Einstein condition

$$
\sqrt{-1} \Lambda_{\omega} F_{H}=\lambda \cdot \operatorname{Id}_{E},
$$

where $\lambda=\frac{2 \pi \mu_{\omega}(E)}{\operatorname{Vol}(X)}$. When the $(1,1)$-form $\omega$ is understood, we omit the subscript $\omega$ in the above definitions.

In this paper, we consider the following Hermitian-Yang-Mills flow on the holomorphic bundle $\left(E, \bar{\partial}_{E}\right)$ with initial data $H(0)=H_{0}$,

$$
H^{-1} \frac{\partial H}{\partial t}=-2\left(\sqrt{-1} \Lambda_{\omega} F_{H}-\lambda \operatorname{Id}_{E}\right)
$$

1991 Mathematics Subject Classification. 53C07, 58E15.

Key words and phrases. Gauduchon, Astheno-Kähler, Hermitian-Yang-Mills flow.

The authors were supported in part by NSF in China, No.11625106, 11571332, 11526212. 
where $\lambda=\frac{2 \pi \mu_{\omega}(E)}{\operatorname{Vol}(X)}$ and $F_{H}$ is the curvature of the Chern connection with respect to $H$. The Hermitian-Yang-Mills flow (1.1) was introduced and studied by Donaldson in [9]. When $(X, \omega)$ is Kähler, Donaldson proved the long time existence and uniqueness of the solution for (1.1). Using this flow, Donaldson([10]) obtained the existence of the irreducible Hermitian-Einstein metrics on stable bundles over algebraic manifolds which was extended by Uhlenbeck and Yau ([23]) to the Kähler case. On general Hermitian manifolds, the second author ([24]) got the long-time existence and uniqueness of the solution of (1.1).

Let's consider the Hermitian vector bundle $\left(E, H_{0}\right)$. Denote the space of connections of $E$ compatible with $H_{0}$ by $\mathcal{A}_{H_{0}}$ and the space of unitary integrable connections of $E$ by $\mathcal{A}_{H_{0}}^{1,1}$. We denote by $\mathbf{G}^{\mathbb{C}}$ (resp. $\mathbf{G}$, where $\mathbf{G}=\left\{\sigma \in \mathbf{G}^{\mathbb{C}} \mid \sigma^{* H_{0}} \sigma=\mathrm{Id}\right\}$ ) the complex gauge group (resp. unitary gauge group) of the Hermitian vector bundle $\left(E, H_{0}\right) . \mathbf{G}^{\mathbb{C}}$ acts on the space $\mathcal{A}_{H_{0}}$ as follows: for $\sigma \in \mathbf{G}^{\mathbb{C}}$ and $A \in \mathcal{A}_{H_{0}}$,

$$
\bar{\partial}_{\sigma(A)}=\sigma \circ \bar{\partial}_{A} \circ \sigma^{-1}, \quad \partial_{\sigma(A)}=\left(\sigma^{* H_{0}}\right)^{-1} \circ \partial_{A} \circ \sigma^{* H_{0}} .
$$

Following Donaldson's argument ([9]), we can show that the Hermitian-Yang-Mills flow (1.1) is gauge equivalent to the following heat flow

$$
\left\{\begin{array}{l}
\frac{\partial A(t)}{\partial t}=\sqrt{-1}\left(\bar{\partial}_{A}-\partial_{A}\right) \Lambda_{\omega} F_{A}, \\
A(0)=A_{0},
\end{array}\right.
$$

where $A_{0}=\left(\bar{\partial}_{E}, H_{0}\right)$. In fact, there is a family of complex gauge transformations $\sigma(t) \in \mathbf{G}^{\mathbb{C}}$ satisfying $\sigma(t)^{* H_{0}} \sigma(t)=h(t)=H_{0}^{-1} H(t)$, where $H(t)$ is the long time solution of the HermitianYang-Mills flow (1.1) with the initial metric $H_{0}$, such that $A(t)=\sigma(t)\left(A_{0}\right)$ is the long time solution of the heat flow (1.3) with the initial connection $A_{0}$.

When the underground manifold $(X, \omega)$ is Kähler, it is easy to see that the heat flow (1.3) is just the Yang-Mills flow by the Kähler identity. There are many interesting results on the convergence of the Yang-Mills flow, see references [1, 3, 4, 6, 7, 13, 17, 20]. In this article, we study the limiting behaviour of the Hermitian-Yang-Mills flow (or the heat flow (1.3)) under the assumption that $\omega$ is Gauduchon and Astheno-Kähler. We first give some basic properties of the heat flow (1.3) including energy inequality, monotonicity formula of certain quantities and small energy regularity. Then, following the argument of Hong and Tian in ([13]) and using Bando and Siu's extension technique, we obtain the following convergence result of the heat flow (1.3)

Theorem 1.1. Let $(X, \omega)$ be an $n$-dimensional compact Hermitian manifold with $\omega$ satisfying $\partial \bar{\partial} \omega^{n-1}=\partial \bar{\partial} \omega^{n-2}=0$. Suppose $A(t)$ is the global smooth solution of the heat flow (1.3) on the Hermitian vector bundle $\left(E, H_{0}\right)$ with the initial data $A_{0}$ over $(X, \omega)$. Then

(1) For every sequence $t_{k} \rightarrow \infty$, there is a subsequence $t_{k_{j}}$ such that as $t_{k_{j}} \rightarrow \infty, A\left(t_{k_{j}}\right)$ converges modulo gauge transformations to a solution $A_{\infty}$ of equation

$$
D_{A} \Lambda_{\omega} F_{A}=0
$$

on Hermitian vector bundle $\left(E_{\infty}, H_{\infty}\right)$ in $C_{\text {loc }}^{\infty}$ topology outside a subset $\Sigma \subset X$, where $\Sigma$ is a closed set of Hausdorff codimension at least 4.

(2) The limiting $\left(E_{\infty}, H_{\infty}, \bar{\partial}_{A_{\infty}}\right)$ can be extended to the whole $X$ as a reflexive sheaf with a holomorphic orthogonal splitting

$$
\left(E_{\infty}, H_{\infty}, A_{\infty}\right)=\bigoplus_{i=1}^{l}\left(E_{\infty}^{i}, H_{\infty}^{i}, A_{\infty}^{i}\right),
$$

where $H_{\infty}^{i}$ is an admissible Hermitian-Einstein metric on reflexive sheaf $E_{\infty}^{i}$. 
The paper is organised as below. In Section 2, we present some basic properties of the heat flow (1.3). In Section 3, we give the detailed proof of Theorem 1.1.

\section{EXIstence OF the HeAT FLOW AND SOME BASIC ESTimates}

Let $(X, \omega)$ be an $n$ dimensional compact Hermitian manifold and $\left(E, H_{0}\right)$ a rank $r$ complex vector bundle over $(X, \omega)$. On the space $\mathcal{A}_{H_{0}}$, we define the Yang-Mills functional by

$$
\mathrm{YM}(\mathrm{A})=\int_{\mathrm{X}}\left|\mathrm{F}_{\mathrm{A}}\right|^{2} \mathrm{~d} \mathrm{~V}_{\omega}
$$

And the negative gradient flow of Yang-Mills functional is

which is called the Yang-Mills flow.

$$
\frac{\partial A}{\partial t}=-D_{A}^{*} F_{A}
$$

Using the Taylor expansion method, Demailly ([8]) showed that for any $A \in \mathcal{A}_{H_{0}}^{1,1}$, it holds

$$
\bar{\partial}_{A}^{*}=-\sqrt{-1}\left[\Lambda_{\omega}, \partial_{A}\right]-\bar{\tau}^{*}, \quad \partial_{A}^{*}=\sqrt{-1}\left[\Lambda_{\omega}, \bar{\partial}_{A}\right]-\tau^{*},
$$

where $\tau=\left[\Lambda_{\omega}, \partial \omega\right]$. From (2.1), we know that the heat flow (1.3) is equivalent to

$$
\left\{\begin{array}{l}
\frac{\partial A(t)}{\partial t}=-D_{A}^{*} F_{A}-\left[\Lambda_{\omega}, d w\right]^{*} F_{A} \\
A(0)=A_{0}
\end{array}\right.
$$

Using the result in [24] and following the argument of Donaldson([9]), we can obtain the long time existence and uniqueness of solution of the heat flow (1.3). Since the proof is similar as that in [9], we omit it.

Theorem 2.1. Let $(X, \omega)$ be an n-dimensional compact Hermitian manifold and $\left(E, H_{0}\right)$ a rank $r$ Hermitian vector bundle over $X$. Given any $A_{0} \in \mathcal{A}_{H_{0}}^{1,1}$, the heat flow (1.3) has a unique long-time solution in the complex gauge orbit of $A_{0}$ with the initial data $A_{0}$.

2.1. Basic estimates. Suppose that $A(t)$ is a smooth solution of the heat flow (1.3) and $f$ a real smooth function over $X$. It holds that

$$
\begin{aligned}
\frac{d}{d t} \int_{X} f^{2}\left|F_{A}\right|^{2} d V_{g}= & 2 \operatorname{Re} \int_{X}\left\langle f^{2} F_{A}, D_{A} \frac{d A}{d t}\right\rangle d V_{g} \\
= & 2 \operatorname{Re}\left\{\int_{X} f^{2}\left\langle D_{A}^{*} F_{A}, \frac{\partial A}{\partial t}\right\rangle d V_{g}-\int_{X}\left\langle F_{A}, d f^{2} \wedge \frac{\partial A}{\partial t}\right\rangle d V_{g}\right\} \\
= & -2 \int_{X} f^{2}\left|\frac{\partial A}{\partial t}\right|^{2} d V_{g}-2 \operatorname{Re} \int_{X} f^{2}\left\langle(\tau+\bar{\tau})^{*} F_{A}, \frac{\partial A}{\partial t}\right\rangle d V_{g} \\
& -2 \operatorname{Re} \int_{X}\left\langle F_{A}, d f^{2} \wedge \frac{\partial A}{\partial t}\right\rangle d V_{g}
\end{aligned}
$$

Setting $f \equiv 1$ on $X$, we get

$$
\frac{d}{d t} \int_{X}\left|F_{A}\right|^{2} d V_{g}=-2 \int_{X}\left|\frac{\partial A}{\partial t}\right|^{2} d V_{g}-2 \operatorname{Re} \int_{X}\left\langle(\tau+\bar{\tau})^{*} F_{A}, \frac{\partial A}{\partial t}\right\rangle d V_{g}
$$

Proposition 2.2. If the fundament form $\omega$ satisfies $\partial \bar{\partial} \omega^{n-1}=\partial \bar{\partial} \omega^{n-2}=0$, there holds

$$
\int_{X}\left\langle(\tau+\bar{\tau})^{*} F_{A}, \frac{\partial A}{\partial t}\right\rangle d V_{g}=0
$$


Proof. From Proposition 4.1 in [19], we have

$$
\tau^{*} F_{A}=-\frac{*\left(\bar{\partial}\left(\omega^{n-2}\right) \wedge F_{A}\right)}{(n-2) !}+\frac{*\left(\bar{\partial}\left(\omega^{n-1}\right) \Lambda_{\omega} F_{A}\right)}{(n-1) !}
$$

and

$$
\bar{\tau}^{*} F_{A}=-\frac{*\left(\partial\left(\omega^{n-2}\right) \wedge F_{A}\right)}{(n-2) !}+\frac{*\left(\partial\left(\omega^{n-1}\right) \Lambda_{\omega} F_{A}\right)}{(n-1) !} .
$$

At first, by (1.3),(2.6) and Stokes formula, we have

$$
\begin{aligned}
& \int_{X}\left\langle\tau^{*} F_{A}, \frac{\partial A}{\partial t}\right\rangle d V_{g} \\
= & \int_{X}\left\langle\tau^{*} F_{A}, \sqrt{-1 \partial_{A}} \Lambda_{\omega} F_{A}\right\rangle d V_{g} \\
= & \int_{X}\left\langle-\frac{*\left(\bar{\partial}\left(\omega^{n-2}\right) \wedge F_{A}\right)}{(n-2) !}+\frac{*\left(\bar{\partial}\left(\omega^{n-1}\right) \Lambda_{\omega} F_{A}\right)}{(n-1) !}, \sqrt{-1 \partial_{A}} \Lambda_{\omega} F_{A}\right\rangle d V_{g} \\
= & \int_{X} \sqrt{-1}\left\langle\frac{\bar{\partial}_{A}^{*} *\left(\bar{\partial}\left(\omega^{n-2}\right) \wedge F_{A}\right)}{(n-2) !}, \Lambda_{\omega} F_{A}\right\rangle d V_{g}-\int_{X} \sqrt{-1}\left\langle\frac{\bar{\partial}_{A}^{*} *\left(\bar{\partial}\left(\omega^{n-1}\right) \Lambda_{\omega} F_{A}\right)}{(n-1) !}, \Lambda_{\omega} F_{A}\right\rangle d V_{g} \\
= & I+I I .
\end{aligned}
$$

Then, by simple calculation, we have

$$
\begin{aligned}
I & =\int_{X} \sqrt{-1}\left\langle\frac{\bar{\partial}_{A}^{*} *\left(\bar{\partial}\left(\omega^{n-2}\right) \wedge F_{A}\right)}{(n-2) !}, \Lambda_{\omega} F_{A}\right\rangle \\
& =\int_{X} \sqrt{-1}\left\langle\frac{* \partial_{A}\left(\bar{\partial}\left(\omega^{n-2}\right) \wedge F_{A}\right)}{(n-2) !}, \Lambda_{\omega} F_{A}\right\rangle \\
& =\int_{X} \sqrt{-1}\left\langle\frac{*\left(\partial \bar{\partial}\left(\omega^{n-2}\right) \wedge F_{A}\right)}{(n-2) !}, \Lambda_{\omega} F_{A}\right\rangle \\
& =0
\end{aligned}
$$

and

$$
\begin{aligned}
I I & =-\int_{X} \sqrt{-1}\left\langle\frac{\bar{\partial}_{A}^{*} *\left(\bar{\partial}\left(\omega^{n-1}\right) \Lambda_{\omega} F_{A}\right)}{(n-1) !}, \Lambda_{\omega} F_{A}\right\rangle d V_{g} \\
& =-\int_{X} \sqrt{-1}\left\langle\frac{* \partial_{A}\left(\bar{\partial}\left(\omega^{n-1}\right) \Lambda_{\omega} F_{A}\right)}{(n-1) !}, \Lambda_{\omega} F_{A}\right\rangle d V_{g} \\
& =-\int_{X} \sqrt{-1}\left\langle\frac{*\left(\partial \bar{\partial}\left(\omega^{n-1}\right) \Lambda_{\omega} F_{A}\right)}{(n-1) !}, \Lambda_{\omega} F_{A}\right\rangle d V_{g}+\int_{X} \sqrt{-1}\left\langle\frac{*\left(\bar{\partial} \omega^{n-1} \wedge \partial_{A} \Lambda_{\omega} F_{A}\right)}{(n-1) !}, \Lambda_{\omega} F_{A}\right\rangle d V_{g} \\
& =-\int_{X} \sqrt{-1} \operatorname{tr}\left\{\Lambda_{\omega} F_{A} \frac{\left(\bar{\partial} \omega^{n-1} \wedge \partial_{A} \Lambda_{\omega} F_{A}\right)}{(n-1) !}\right\} \\
& =-\int_{X} \frac{\sqrt{-1}}{2} \partial\left|\Lambda_{\omega} F_{A}\right|^{2} \wedge \frac{\bar{\partial} \omega^{n-1}}{(n-1) !} \\
& =0 .
\end{aligned}
$$

In the same way, we have

$$
\int_{X}\left\langle\bar{\tau}^{*} F_{A}, \frac{\partial A}{\partial t}\right\rangle d V_{g}=0
$$


Therefore, there holds that

Lemma 2.3. Let $A(t)$ be a solution of the heat flow (1.3) with initial data $A_{0}$ over $X$. Then

$$
\mathrm{YM}(t)+2 \int_{0}^{t} \int_{X}\left|\frac{\partial A}{\partial t}\right|^{2}=\mathrm{YM}(0)
$$

Let $f$ be a cut-off function with support inside $B_{2 R}\left(x_{0}\right)$ and $f \equiv 1$ on $B_{R}\left(x_{0}\right)$ such that $0 \leq f \leq 1$ and $|d f| \leq 2 R^{-1}$. Set $e(A)=\left|F_{A}\right|^{2}$. From the identity (2.3), we have

$$
\left.\left|\frac{d}{d t} \int_{X} f^{2} e(A)+2 \int_{X} f^{2}\right| \frac{\partial A}{\partial t}\right|^{2} \mid \leq C\left(\int_{X} f|d f|\left|F_{A}\right|\left|\frac{\partial A}{\partial t}\right|+\int_{X} f^{2}\left|F_{A}\right|\left|\frac{\partial A}{\partial t}\right|\right)
$$

Then from (2.9), we can deduce the following local energy estimates:

Lemma 2.4. (Local energy estimates) Suppose $A(t)$ is a smooth solution of the heat flow (2.2). Fix $x_{0} \in X$ and $R \in \mathbb{R}^{+}$such that $B_{2 R}\left(x_{0}\right) \subset X$. Then for any two finite numbers $s$ and $\tau$, we have

$$
\begin{aligned}
\int_{B_{R}\left(x_{0}\right)} e(A)(\cdot, s) d V_{g} \leq & \int_{B_{2 R}\left(x_{0}\right)} e(A)(\cdot, \tau) d V_{g}+2 \int_{\min \{s, \tau\}}^{\max \{s, \tau\}} \int_{X}\left|\frac{\partial A}{\partial t}\right|^{2} d V_{g} d t \\
& +C\left(\frac{|s-\tau|}{R^{2}} \mathrm{YM}(0) \int_{\min \{\mathrm{s}, \tau\}}^{\max \{\mathrm{s}, \tau\}} \int_{\mathrm{X}}\left|\frac{\partial \mathrm{A}}{\partial \mathrm{t}}\right|^{2} \mathrm{dV} \mathrm{g} \mathrm{dt}\right)^{\frac{1}{2}} \\
& +\left(C|s-\tau| \mathrm{YM}(0) \int_{\min \{\mathrm{s}, \tau\}}^{\max \{\mathrm{s}, \tau\}} \int_{\mathrm{X}}\left|\frac{\partial \mathrm{A}}{\partial \mathrm{t}}\right|^{2}\right)^{\frac{1}{2}} .
\end{aligned}
$$

Proof. From (2.9) and Hölder inequality, we have

$$
\begin{aligned}
& \left|\int_{\min \{s, \tau\}}^{\max \{s, \tau\}}\left(\frac{d}{d t} \int_{X} f^{2} e(A)+2 \int_{X} f^{2}\left|\frac{\partial A}{\partial t}\right|^{2}\right)\right| \\
\leq & \left.\int_{\min \{s, \tau\}}^{\max \{s, \tau\}}\left|\frac{d}{d t} \int_{X} f^{2} e(A)+2 \int_{X} f^{2}\right| \frac{\partial A}{\partial t}\right|^{2} \mid \\
\leq & C / R \int_{\min \{s, \tau\}}^{\max \{s, \tau\}}\left(\int_{X} f^{2} e(A) d V_{g}\right)^{\frac{1}{2}}\left(\int_{X}\left|\frac{\partial A}{\partial t}\right|^{2}\right)^{\frac{1}{2}} \\
& +C \int_{\min \{s, \tau\}}^{\max \{s, \tau\}}\left(\int_{X} f^{2} e(A) d V_{g}\right)^{\frac{1}{2}}\left(\int_{X}\left|\frac{\partial A}{\partial t}\right|^{2}\right)^{\frac{1}{2}} \\
\leq & \left(\frac{C|s-\tau|}{R^{2}} \mathrm{YM}(0) \int_{\min \{\mathrm{s}, \tau\}}^{\max \{\mathrm{s}, \tau\}} \int_{\mathrm{X}}\left|\frac{\partial \mathrm{A}}{\partial \mathrm{t}}\right|^{2}\right)^{\frac{1}{2}} \\
& +\left(C|s-\tau| \mathrm{YM}(0) \int_{\min \{s, \tau\}}^{\max \{s, \tau\}} \int_{X}\left|\frac{\partial A}{\partial t}\right|^{2}\right)^{\frac{1}{2}} .
\end{aligned}
$$


This indicates that

$$
\begin{aligned}
& \int_{B_{R}\left(x_{0}\right)} e(A)(\cdot, s) d V_{g} \\
\leq & \int_{B_{2 R}\left(x_{0}\right)} e(A)(\cdot, \tau) d V_{g}+2 \int_{\min \{s, \tau\}}^{\max \{s, \tau\}} \int_{X}\left|\frac{\partial A}{\partial t}\right|^{2} d V_{g} d t \\
& +C\left(\frac{|s-\tau|}{R^{2}} Y M(0) \int_{\min \{s, \tau\}}^{\max \{s, \tau\}} \int_{X}\left|\frac{\partial A}{\partial t}\right|^{2} d V_{g} d t\right)^{\frac{1}{2}} \\
& +\left(C|s-\tau| \mathrm{YM}(0) \int_{\min \{s, \tau\}}^{\max \{s, \tau\}} \int_{X}\left|\frac{\partial A}{\partial t}\right|^{2}\right)^{\frac{1}{2}} .
\end{aligned}
$$

Lemma 2.5. Suppose $A(t)$ is a smooth solution of the heat flow (1.3). Then it holds that

$$
\left(\frac{\partial}{\partial t}-2 \sqrt{-1} \Lambda_{\omega} \partial \bar{\partial}\right)\left|\Lambda_{\omega} F_{A}\right|^{2}=-2\left|D_{A} \Lambda_{\omega} F_{A}\right|^{2} \leq 0 .
$$

Furthermore, $\left\|\Lambda_{\omega} F_{A}\right\|_{L^{2}}^{2}(t)$ is decreasing along the flow and the $L^{\infty}$ norm of the $\Lambda_{\omega} F_{A}$ is bounded.

Proof. By simple calculation, we have

$$
\begin{aligned}
\frac{\partial}{\partial t}\left|\Lambda_{\omega} F_{A}\right|^{2} & =2 \operatorname{Re}\left\langle\frac{\partial}{\partial t} \Lambda_{\omega} F_{A}, \Lambda_{\omega} F_{A}\right\rangle \\
& =2 \operatorname{Re} \sqrt{-1} \Lambda_{\omega}\left\langle\left(\partial_{A} \bar{\partial}_{A}-\bar{\partial}_{A} \partial_{A}\right) \Lambda_{\omega} F_{A}, \Lambda_{\omega} F_{A}\right\rangle
\end{aligned}
$$

and

$$
\begin{aligned}
\sqrt{-1} \Lambda_{\omega} \partial \bar{\partial}\left|\Lambda_{\omega} F_{A}\right|^{2}= & \sqrt{-1} \Lambda_{\omega} \partial\left\langle\bar{\partial}_{A} \Lambda_{\omega} F_{A}, \Lambda_{\omega} F_{A}\right\rangle+\sqrt{-1} \Lambda_{\omega} \partial\left\langle\Lambda_{\omega} F_{A}, \partial_{A} \Lambda_{\omega} F_{A}\right\rangle \\
= & \sqrt{-1} \Lambda_{\omega}\left\langle\partial_{A} \bar{\partial}_{A} \Lambda_{\omega} F_{A}, \Lambda_{\omega} F_{A}\right\rangle+\left|\bar{\partial}_{A} \Lambda_{\omega} F_{A}\right|^{2}+\left|\partial_{A} \Lambda_{\omega} F_{A}\right|^{2} \\
& +\sqrt{-1} \Lambda_{\omega}\left\langle\Lambda_{\omega} F_{A}, \bar{\partial}_{A} \partial_{A} \Lambda_{\omega} F_{A}\right\rangle \\
& =\operatorname{Re} \sqrt{-1} \Lambda_{\omega}\left\langle\left(\partial_{A} \bar{\partial}_{A}-\bar{\partial}_{A} \partial_{A}\right) \Lambda_{\omega} F_{A}, \Lambda_{\omega} F_{A}\right\rangle+\left|D_{A} \Lambda_{\omega} F_{A}\right|^{2}
\end{aligned}
$$

This implies that

$$
\left(\frac{\partial}{\partial t}-2 \sqrt{-1} \Lambda_{\omega} \partial \bar{\partial}\right)\left|\Lambda_{\omega} F_{A}\right|^{2}=-2\left|D_{A} \Lambda_{\omega} F_{A}\right|^{2} \leq 0 .
$$

Using the maximum principle, we have

$$
\sup _{X}\left|\Lambda_{\omega} F_{A}\right|^{2}(\cdot, t) \leq \sup _{X}\left|\Lambda_{\omega} F_{A}\right|^{2}(\cdot, 0) .
$$

Integrating the two sides of (2.10) over $X$, we have

$$
\begin{aligned}
& \frac{\partial}{\partial t} \int_{X}\left|\Lambda_{\omega} F_{A}\right|^{2} \frac{\omega^{n}}{n !}-2 \int_{X} \sqrt{-1} \Lambda_{\omega} \partial \bar{\partial}\left|\Lambda_{\omega} F_{A}\right|^{2} \frac{\omega^{n}}{n !} \\
= & \frac{\partial}{\partial t} \int_{X}\left|\Lambda_{\omega} F_{A}\right|^{2} \frac{\omega^{n}}{n !}-2 \int_{X} \sqrt{-1}\left|\Lambda_{\omega} F_{A}\right|^{2} \frac{\bar{\partial} \partial \omega^{n-1}}{(n-1) !} \\
= & \frac{\partial}{\partial t} \int_{X}\left|\Lambda_{\omega} F_{A}\right|^{2} \frac{\omega^{n}}{n !}=-\int_{X}\left|D_{A} \Lambda_{\omega} F_{A}\right|^{2} \frac{\omega^{n}}{n !} \leq 0 .
\end{aligned}
$$

Therefore, $\left\|\Lambda_{\omega} F_{A}\right\|_{L^{2}}^{2}(t)$ is decreasing along the flow. 
Lemma 2.6. Suppose $A(t)$ is a smooth solution of (1.3) and set

$$
I(t)=\int_{X}\left|D_{A} \Lambda_{\omega} F_{A}\right|^{2}
$$

Then it holds that $I(t) \rightarrow 0$ as $t \rightarrow \infty$.

Proof. From equation (2.2), we have

$$
\begin{aligned}
\frac{d}{d t} D_{A} \Lambda_{\omega} F_{A} & =\left[\frac{\partial A}{\partial t}, \Lambda_{\omega} F_{A}\right]+D_{A} \Lambda_{\omega} \frac{\partial F_{A}}{\partial t} \\
& =\left[\sqrt{-1}\left(\bar{\partial}_{A}-\partial_{A}\right) \Lambda_{\omega} F_{A}, \Lambda_{\omega} F_{A}\right]+D_{A} \Lambda_{\omega} D_{A} \frac{\partial A}{\partial t} \\
& =\left[\sqrt{-1}\left(\bar{\partial}_{A}-\partial_{A}\right) \Lambda_{\omega} F_{A}, \Lambda_{\omega} F_{A}\right]+D_{A} \Lambda_{\omega} D_{A} \sqrt{-1}\left(\bar{\partial}_{A}-\partial_{A}\right) \Lambda_{\omega} F_{A}
\end{aligned}
$$

So

$$
\begin{aligned}
\frac{d}{d t} I(t)= & 2 \operatorname{Re} \int_{X}\left\langle\frac{d}{d t}\left(D_{A} \Lambda_{\omega} F_{A}\right), D_{A} \Lambda_{\omega} F_{A}\right\rangle \\
= & 2 \operatorname{Re} \int_{X}\left\langle\left[\sqrt{-1}\left(\bar{\partial}_{A}-\partial_{A}\right) \Lambda_{\omega} F_{A}, \Lambda_{\omega} F_{A}\right], D_{A} \Lambda_{\omega} F_{A}\right\rangle \\
& +2 \operatorname{Re} \int_{X}\left\langle D_{A} \Lambda_{\omega} D_{A} \sqrt{-1}\left(\bar{\partial}_{A}-\partial_{A}\right) \Lambda_{\omega} F_{A}, D_{A} \Lambda_{\omega} F_{A}\right\rangle \\
= & 2 \operatorname{Re} \int_{X}\left\langle\left[\sqrt{-1}\left(\bar{\partial}_{A}-\partial_{A}\right) \Lambda_{\omega} F_{A}, \Lambda_{\omega} F_{A}\right], D_{A} \Lambda_{\omega} F_{A}\right\rangle \\
& -2 \int_{X}\left|D_{A}^{*} D_{A} \Lambda_{\omega} F_{A}\right|^{2}-2 \operatorname{Re} \int_{X}\left\langle(\tau+\bar{\tau})^{*} D_{A} \Lambda_{\omega} F_{A}, D_{A}^{*} D_{A} \Lambda_{\omega} F_{A}\right\rangle .
\end{aligned}
$$

At first, one can easily check that

$$
\operatorname{Re} \int_{X}\left\langle\left[\sqrt{-1}\left(\bar{\partial}_{A}-\partial_{A}\right) \Lambda_{\omega} F_{A}, \Lambda_{\omega} F_{A}\right], D_{A} \Lambda_{\omega} F_{A}\right\rangle \leq C\left(n, \operatorname{rank} E,\left\|\Lambda_{\omega} F_{A}\right\|_{L^{\infty}}\right) I(t) .
$$

Then, it holds

$$
\begin{aligned}
\int_{X}\left\langle D_{A} \Lambda_{\omega} F_{A}, D_{A} \Lambda_{\omega} F_{A}\right\rangle & =\int_{X}\left\langle\Lambda_{\omega} F_{A}, D_{A}^{*} D_{A} \Lambda_{\omega} F_{A}\right\rangle \\
& \leq\left\|\Lambda_{\omega} F_{A}\right\|_{L^{\infty}} \int_{X}\left|D_{A}^{*} D_{A} \Lambda_{\omega} F_{A}\right| \\
& \leq\left\|\Lambda_{\omega} F_{A}\right\|_{L^{\infty}} \operatorname{Vol}(X)^{1 / 2}\left(\int_{X}\left|D_{A}^{*} D_{A} \Lambda_{\omega} F_{A}\right|^{2}\right)^{1 / 2}
\end{aligned}
$$

Inequality (2.11) implies

$$
I(t)^{2} \leq\left\|\Lambda_{\omega} F_{A}\right\|_{L^{\infty}}^{2} \operatorname{Vol}(X) \int_{X}\left|D_{A}^{*} D_{A} \Lambda_{\omega} F_{A}\right|^{2} .
$$

At last, it is easy to check that

$$
\begin{aligned}
\int_{X}\left\langle(\tau+\bar{\tau})^{*} D_{A} \Lambda_{\omega} F_{A}, D_{A}^{*} D_{A} \Lambda_{\omega} F_{A}\right\rangle & =\int_{X}\left\langle D_{A} \Lambda_{\omega} F_{A}, \Lambda_{\omega} d \omega \wedge D_{A}^{*} D_{A} \Lambda_{\omega} F_{A}\right\rangle \\
& \leq \int_{X}\left|D_{A}^{*} D_{A} \Lambda_{\omega} F_{A}\right|^{2}+C^{2} I(t) .
\end{aligned}
$$


From the above all, we have

$$
\begin{aligned}
\frac{d I(t)}{d t} & \leq C I(t)-\int_{X}\left|D_{A}^{*} D_{A} \Lambda_{\omega} F_{A}\right|^{2}+C^{2} I(t) \\
& \leq C I(t)-C I(t)^{2} .
\end{aligned}
$$

From equality (2.9) and

$$
\int_{X}\left|\frac{\partial A}{\partial t}\right|^{2}=\int_{X}\left|\left(\bar{\partial}_{A}-\partial_{A}\right) \Lambda_{\omega} F_{A}\right|^{2}=\int_{X}\left|D_{A} \Lambda_{\omega} F_{A}\right|^{2}
$$

we have

$$
\int_{0}^{\infty} I(t)<Y M(0)
$$

Using the technique in [11, Prop. 6.2.14], we have $I(t) \rightarrow 0$ as $t \rightarrow \infty$.

Lemma 2.7. Suppose $A(t)$ is a global smooth solution of heat flow (1.3). Then it holds that

$$
\left(\triangle-\frac{\partial}{\partial t}\right)\left|F_{A}\right|^{2} \geq 2\left|\nabla_{A} F_{A}\right|^{2}-C\left(1+\left|F_{A}\right|+|R i c|+|R m|\right)\left|F_{A}\right|^{2}-C\left|F_{A}\right|\left|\nabla_{A} F_{A}\right|,
$$

where $C$ is a positive constant depending on the geometry of $X$.

Proof. First, using Bochner technique, we have

$$
\triangle\left|F_{A}\right|^{2}=-2\left\langle\nabla_{A}^{*} \nabla_{A} F_{A}, F_{A}\right\rangle+2\left|\nabla_{A} F_{A}\right|^{2} .
$$

Then by simple calculation, we have

$$
\frac{\partial}{\partial t} F_{A}=D_{A} \frac{\partial A}{\partial t}=-D_{A} D_{A}^{*} F_{A}-D_{A} \alpha
$$

where $\alpha=\left[\Lambda_{\omega}, d \omega\right]^{*} F_{A}$. Combining with the following Weitzenböck formula

$$
\triangle_{A} F_{A}=-D_{A} D_{A}^{*} F_{A}=\nabla_{A}^{*} \nabla_{A} F_{A}+R i c \sharp F_{A}+F_{A} \sharp F_{A},
$$

we have

Therefore,

$$
\frac{\partial}{\partial t} F_{A}=-\nabla_{A}^{*} \nabla_{A} F_{A}-R i c \sharp F_{A}-F_{A} \sharp F_{A}-D_{A} \alpha .
$$

$$
\begin{aligned}
\left(\triangle-\frac{\partial}{\partial t}\right)\left|F_{A}\right|^{2} & =2\left|\nabla_{A} F_{A}\right|^{2}+2\left\langle R i c \sharp F_{A}+F_{A} \sharp F_{A}+D_{A} \alpha, F_{A}\right\rangle \\
& \geq 2\left|\nabla_{A} F_{A}\right|^{2}-C\left(1+|R i c|+\left|F_{A}\right|\right)\left|F_{A}\right|^{2}-C\left|\nabla_{A} F_{A}\right|\left|F_{A}\right|,
\end{aligned}
$$

where $C$ is a constant depending on the geometry of $X$.

2.2. Monotonicity formula. Let $(X, g)$ be an $n$-dimensional compact Hermitian manifold with fundamental $(1,1)$-form $\omega$ satisfying $\partial \bar{\partial} \omega^{n-1}=\partial \bar{\partial} \omega^{n-2}=0$. We regard $X$ as a $2 n$-dimensional Riemannian manifold. For any $x_{0} \in X$, there exist normal geodesic coordinates $\left\{x_{i}\right\}_{i=1}^{2 n}$ in the geodesic ball $B_{r}\left(x_{0}\right)$ centered at $x_{0}$ with radius $r \leq i_{X}$ such that $x_{0}=(0, \ldots, 0)$ and

$$
\left|g_{i j}(x)-\delta_{i j}\right| \leq C\left(x_{0}\right)|x|^{2}, \quad\left|\frac{\partial g_{i j}}{\partial x_{k}}\right| \leq C\left(x_{0}\right)|x| \quad \forall x \in B_{r}
$$

where $i(X)$ is the infimum of the injectivity radius over $X$ and $C\left(x_{0}\right)$ a positive constant depending on $x_{0}$. 
Let $u=(x, t)$ be a point in $X \times \mathbb{R}$. For a fixed point $u_{0}=\left(x_{0}, t_{0}\right) \in X \times \mathbb{R}^{+}$, we write

$$
\begin{aligned}
& S_{r}=X \times\left\{t=t_{0}-r^{2}\right\} \\
& T_{r}=\left\{u=(x, t): t_{0}-4 r^{2} \leq t \leq t_{0}-r^{2}, x \in X\right\} \\
& P_{r}\left(u_{0}\right)=B_{r}\left(x_{0}\right) \times\left[t_{0}-r^{2}, t_{0}+r^{2}\right] .
\end{aligned}
$$

For simplicity, we denote $S_{r}(0,0), T_{r}(0,0)$ and $P_{r}(0,0)$ by $S_{r}, T_{r}$ and $P_{r}$ respectively.

The fundamental solution of the (backward) heat equation with singularity at $\left(x_{0}, t_{0}\right)$ is

$$
G_{\left(x_{0}, t_{0}\right)}(x, t)=\frac{1}{\left(4 \pi\left(t_{0}-t\right)\right)^{2 n}} \exp \left(-\frac{\left|x-x_{0}\right|^{2}}{4\left(t_{0}-t\right)}\right), \quad t \leq t_{0} .
$$

For simplicity, we denote $G_{(0,0)}(x, t)$ by $G(x, t)$.

Assume that $A(t)$ is a smooth global solution of the heat flow (1.3) in $X \times \mathbb{R}^{+}$. Let $f$ be a smooth cut-off function such that $|f| \leq 1, f \equiv 1$ on $B_{R / 2}, f=0$ outside $B_{R}$ and $|\nabla f| \leq 2 / R$, where $R \leq i_{X}$. For any $(x, t) \in X \times[0,+\infty)$, we set

$$
\Phi(r)=r^{2} \int_{T_{r}\left(u_{0}\right)} e(A) f^{2} G_{u_{0}} d t
$$

Then we have

Theorem 2.8. Assume that $A(t)$ is a solution of the heat flow (1.3) in $X \times R_{+}$with initial data $A_{0}$. Let $f$ be a smooth cut-off function such that $|f| \leq 1, f \equiv 1$ on $B_{R / 2}, f=0$ outside $B_{R}$ and $|\nabla f| \leq 2 / R$, where $R \leq i(X)$. Then for any $r_{1}$ and $r_{2}$ with $0<r_{1} \leq r_{2} \leq \min \left\{R / 2, \sqrt{t_{0}} / 2\right\}$, we have

$$
\Phi\left(r_{1}\right) \leq C \exp \left(C\left(r_{2}-r_{1}\right)\right) \Phi\left(r_{2}\right)+C\left(r_{2}^{2}-r_{1}^{2}\right) Y M(0)+C R^{2-2 n} \int_{P_{R}\left(u_{0}\right)}\left|F_{A}\right|^{2} d V_{g} d t .
$$

Proof. Choose normal geodesic coordinates $\left\{x_{i}\right\}_{i=1}^{2 n}$ in the geodesic ball $B_{R}\left(x_{0}\right)$. Setting $x=r \widetilde{x}$, $t=t_{0}+r^{2} \widetilde{t}$, we have

$$
\begin{aligned}
\Phi(r) & =r^{2} \int_{T_{r}\left(u_{0}\right)} e(A) f^{2} G_{u_{0}} d V_{g} d t \\
& =r^{2} \int_{t_{0}-4 r^{2}}^{t_{0}-r^{2}} \int_{\mathbb{R}^{2 n}} e(A)(x, t) f^{2}(x) G_{u_{0}}(x, t) \operatorname{det}\left(g_{i j}\right) d x d t \\
& =r^{4} \int_{\tilde{T}_{1}} e(A)\left(r \tilde{x}, t_{0}+r^{2} \tilde{t}\right) f^{2}(r \tilde{x}) G(\tilde{x}, \tilde{t}) \operatorname{det}\left(g_{i j}\right)(r \tilde{x}) d \tilde{x} d \tilde{t}
\end{aligned}
$$

where $\tilde{T}_{1}=[-4,-1] \times \mathbb{R}^{2 n}$. The $r$-direction derivative of $\Phi(r)$ is

$$
\begin{aligned}
d \Phi(r) / d r= & 4 r^{3} \int_{\tilde{T}_{1}} e(A)\left(r \tilde{x}, t_{0}+r^{2} \tilde{t}\right) f^{2}(r \tilde{x}) G(\tilde{x}, \tilde{t}) \operatorname{det} g_{i j}(r \tilde{x}) d \tilde{x} d \tilde{t} \\
& +r^{4} \int_{\tilde{T}_{1}}\left(\frac{d}{d r} e(A)\left(r \tilde{x}, t_{0}+r^{2} \tilde{t}\right)\right) f^{2}(r \tilde{x}) G(\tilde{x}, \tilde{t}) \operatorname{det} g_{i j}(r \tilde{x}) d \tilde{x} d \tilde{t} \\
& +\int_{\tilde{T}_{1}} e(A)\left(r \tilde{x}, t_{0}+r^{2} \tilde{t}\right)\left(\frac{d}{d r} f^{2}(r \tilde{x}) \operatorname{det}\left(g_{i j}\right)(r \tilde{x})\right) G(\tilde{x}, \tilde{t}) d \tilde{x} d \tilde{t} \\
=: & I_{1}+I_{2}+I_{3},
\end{aligned}
$$


where $I_{1}=\frac{4 \Phi(r)}{r}$. At first, we calculate $I_{2}$. By simple calculation, we have

$$
\begin{aligned}
\frac{\partial}{\partial r} e(A)\left(r \tilde{x}^{i}, t_{0}+r^{2} \tilde{t}\right) & =\frac{\partial}{\partial x^{i}} e(A)\left(r \tilde{x}^{i}, t_{0}+r^{2} \tilde{t}\right) \frac{\partial x^{i}}{\partial r}+\frac{\partial}{\partial t} e(A)\left(r \tilde{x}^{i}, t_{0}+r^{2} \tilde{t}\right) \frac{\partial t}{\partial r} \\
& =\tilde{x}^{i} \frac{\partial}{\partial x^{i}} e(A)+2 r \tilde{t} \frac{\partial}{\partial t} e(A) \\
& =\frac{x^{i}}{r} \frac{\partial}{\partial x^{i}} e(A)+\frac{2\left(t-t_{0}\right)}{r} \frac{\partial}{\partial t} e(A) .
\end{aligned}
$$

Substituting (2.12) into $I_{2}$, we have

$$
\begin{aligned}
I_{2}= & r \int_{T_{r}\left(u_{0}\right)}\left(x^{i} \frac{d}{d x^{i}} e(A)(x, t)\right) f^{2}(x) G_{u_{0}}(x, t) d V_{g} d t \\
& +r \int_{T_{r}\left(u_{0}\right)}\left(2\left(t-t_{0}\right) \frac{d}{d t} e(A)(x, t)\right) f^{2}(x) G_{u_{0}}(x, t) d V_{g} d t \\
= & : I_{2.1}+I_{2.2}
\end{aligned}
$$

From the Bianchi identity

$$
D_{A} F_{A}=0
$$

we have

$$
\begin{aligned}
0 & =D_{A} F_{A}\left(\frac{\partial}{\partial x^{i}}, \frac{\partial}{\partial x^{j}}, \frac{\partial}{\partial x^{k}}\right) \\
& =\nabla_{A, \partial / \partial x^{i}} F_{A}\left(\frac{\partial}{\partial x^{j}}, \frac{\partial}{\partial x^{k}}\right)-\nabla_{A, \partial / \partial x^{j}} F_{A}\left(\frac{\partial}{\partial x^{i}}, \frac{\partial}{\partial x^{k}}\right)+\nabla_{A, \partial / \partial x^{k}} F_{A}\left(\frac{\partial}{\partial x^{i}}, \frac{\partial}{\partial x^{j}}\right) .
\end{aligned}
$$

For simplicity, we set $\nabla_{A, i}:=\nabla_{A, \frac{\partial}{\partial x^{i}}}$ and $\nabla_{i}:=\nabla_{\frac{\partial}{\partial x^{i}}}$. Therefore, by (2.13), we have

$$
\begin{aligned}
& x^{i} \frac{\partial}{\partial x^{i}}\left|F_{A}\right|^{2} \\
= & 2 x^{i} \operatorname{Re}\left\langle\nabla_{A, i} F_{A}, F_{A}\right\rangle \\
= & x^{i} \operatorname{Re}\left\langle\nabla_{A, i} F_{A}\left(\frac{\partial}{\partial x^{j}}, \frac{\partial}{\partial x^{k}}\right) d x^{j} \wedge d x^{k}, F_{A}\right\rangle \\
= & x^{i} \operatorname{Re}\left\langle\left(\nabla_{A, j} F_{A}\left(\frac{\partial}{\partial x^{i}}, \frac{\partial}{\partial x^{k}}\right)-\nabla_{A, k} F_{A}\left(\frac{\partial}{\partial x^{i}}, \frac{\partial}{\partial x^{j}}\right)\right) d x^{j} \wedge d x^{k}, F_{A}\right\rangle \\
= & 2 x^{i} \operatorname{Re}\left\langle\nabla_{A, j} F_{A}\left(\frac{\partial}{\partial x^{i}}, \frac{\partial}{\partial x^{k}}\right) d x^{j} \wedge d x^{k}, F_{A}\right\rangle \\
= & 2 \operatorname{Re}\left\langle\left(\nabla_{A, j} x^{i} F_{A}\right)\left(\frac{\partial}{\partial x^{i}}, \frac{\partial}{\partial x^{k}}\right) d x^{j} \wedge d x^{k}, F_{A}\right\rangle-2 \operatorname{Re}\left\langle\delta_{i}^{j} F_{A}\left(\frac{\partial}{\partial x^{i}}, \frac{\partial}{\partial x^{k}}\right) d x^{j} \wedge d x^{k}, F_{A}\right\rangle \\
= & 2 \operatorname{Re}\left\langle d x^{j} \wedge\left(\nabla_{A, j} x^{i} F_{A}\right)\left(\frac{\partial}{\partial x^{i}}, \frac{\partial}{\partial x^{k}}\right) d x^{k}, F_{A}\right\rangle-4\left|F_{A}\right|^{2}
\end{aligned}
$$


and

$$
\begin{aligned}
& d x^{j} \wedge\left(\nabla_{A, j}\left(x^{i} F_{A}\right)\right)\left(\frac{\partial}{\partial x^{i}}, \frac{\partial}{\partial x^{k}}\right) d x^{k} \\
= & d x^{j} \wedge\left(\nabla_{A, j}\left(x^{i} F_{A, i k}\right)-x^{i} F_{A}\left(\nabla_{j} \frac{\partial}{\partial x^{i}}, \frac{\partial}{\partial x^{k}}\right)-x^{i} F_{A}\left(\frac{\partial}{\partial x^{i}}, \nabla_{j} \frac{\partial}{\partial x^{k}}\right)\right) d x^{k} \\
= & \nabla_{A, j}\left(x^{i} F_{A, i k}\right) d x^{j} \wedge d x^{k}-x^{i} F_{A}\left(\nabla_{j} \frac{\partial}{\partial x^{i}}, \frac{\partial}{\partial x^{k}}\right) d x^{j} \wedge d x^{k} \\
= & d x^{j} \wedge \nabla_{A, j}\left(x^{i} F_{A, i k} d x^{k}\right)-x^{i} F_{A, i k} d x^{j} \wedge \nabla_{j} d x^{k}-x^{i} F_{A}\left(\nabla_{j} \frac{\partial}{\partial x^{i}}, \frac{\partial}{\partial x^{k}}\right) d x^{j} \wedge d x^{k} \\
= & D_{A}\left(x^{i} F_{A, i k} d x^{k}\right)-x^{i} F_{A}\left(\nabla_{j} \frac{\partial}{\partial x^{i}}, \frac{\partial}{\partial x^{k}}\right) d x^{j} \wedge d x^{k} .
\end{aligned}
$$

The reason for the second equality in (2.15) is that

$$
\begin{aligned}
& \sum_{j, k} F_{A}\left(\frac{\partial}{\partial x^{i}}, \nabla_{j} \frac{\partial}{\partial x^{k}}\right) d x^{j} \wedge d x^{k} \\
= & \left(\sum_{j<k}+\sum_{k<j}\right) F_{A}\left(\frac{\partial}{\partial x^{i}}, \nabla_{j} \frac{\partial}{\partial x^{k}}\right) d x^{j} \wedge d x^{k} \\
= & \sum_{j<k}\left(F_{A}\left(\frac{\partial}{\partial x^{i}}, \nabla_{j} \frac{\partial}{\partial x^{k}}\right) d x^{j} \wedge d x^{k}+F_{A}\left(\frac{\partial}{\partial x^{i}}, \nabla_{k} \frac{\partial}{\partial x^{j}}\right) d x^{k} \wedge d x^{j}\right) \\
= & 0 .
\end{aligned}
$$

And the reason for the forth equality in (2.15) is that $d x^{j} \wedge \nabla_{j} d x^{k}=D d x^{k}=0$. Substituting (2.15) into (2.14), we have

$$
x^{i} \frac{\partial}{\partial x^{i}}\left|F_{A}\right|^{2}=2 \operatorname{Re}\left\langle D_{A}\left(x^{i} F_{A, i k} d x^{k}\right)-x^{i} F_{A}\left(\nabla_{j} \frac{\partial}{\partial x^{i}}, \frac{\partial}{\partial x^{k}}\right) d x^{j} \wedge d x^{k}, F_{A}\right\rangle-4\left|F_{A}\right|^{2} .
$$

Noting that $\frac{\partial G_{u_{0}}}{\partial x^{\alpha}}=\frac{x^{\alpha} G_{u_{0}}}{2\left(t-t_{0}\right)}$, we have

$$
\begin{aligned}
& \int_{T_{r}\left(u_{0}\right)}\left\langle d\left(f^{2} G_{u_{0}}\right) \wedge\left(x^{i} F_{A, i k} d x^{k}\right), F_{A}\right\rangle d V_{g} d t \\
= & \int_{T_{r}\left(u_{0}\right)}\left\langle x^{i} F_{A, i k} d x^{k}, \nabla\left(f^{2} G_{u_{0}}\right)\left\llcorner F_{A}\right\rangle d V_{g} d t\right. \\
= & \int_{T_{r}\left(u_{0}\right)}\left\langle x^{i} F_{A, i k} d x^{k}, 2 f g^{\alpha \beta} \frac{\partial f}{\partial x^{\alpha}} F_{A, \beta l} d x^{l}\right\rangle G_{u_{0}} d V_{g} d t \\
& +\int_{T_{r}\left(u_{0}\right)}\left\langle x^{i} F_{A, i k} d x^{k}, \frac{g^{\alpha \beta} x^{\alpha}}{2\left(t-t_{0}\right)} F_{A, \beta l} d x^{l}\right\rangle f^{2} G_{u_{0}} d V_{g} d t
\end{aligned}
$$

and

$$
\begin{aligned}
& \int_{T_{r}\left(u_{0}\right)}\left(t-t_{0}\right)\left\langle F_{A}, d\left(f^{2} G_{u_{0}}\right) \wedge \frac{\partial A}{\partial t}\right\rangle d V_{g} d t \\
= & \int_{T_{r}\left(u_{0}\right)}\left(t-t_{0}\right)\left\langle 2 g^{\alpha \beta} \frac{\partial f}{\partial x^{\alpha}} F_{A, \beta l} d x^{l}, \frac{\partial A}{\partial t}\right\rangle f G_{u_{0}} d V_{g} d t \\
& +\int_{T_{r}\left(u_{0}\right)}\left(t-t_{0}\right)\left\langle\frac{g^{\alpha \beta} x^{\alpha}}{2\left(t-t_{0}\right)} F_{A, \beta l} d x^{l}, \frac{\partial A}{\partial t}\right\rangle f^{2} G_{u_{0}} d V_{g} d t .
\end{aligned}
$$


From the above all, we obtain

$$
\begin{aligned}
I_{2.1}= & r \operatorname{Re} \int_{T_{r}\left(u_{0}\right)}\left\langle D_{A}\left(x^{i} F_{A, i k} d x^{k}\right), F_{A}\right\rangle f^{2} G_{u_{0}} d V_{g} d t \\
& -r 2 \operatorname{Re} \int_{T_{r}\left(u_{0}\right)}\left\langle x^{i} F_{A}\left(\nabla_{j} \frac{\partial}{\partial x^{i}}, \frac{\partial}{\partial x^{k}}\right) d x^{j} \wedge d x^{k}, F_{A}\right\rangle f^{2} G_{u_{0}} d V_{g} d t \\
& -4 r \int_{T_{r}\left(u_{0}\right)}\left|F_{A}\right|^{2} f^{2} G_{u_{0}} d V_{g} d t \\
= & -r 2 \operatorname{Re} \int_{T_{r}\left(u_{0}\right)}\left\langle\left(x^{i} F_{A, i k} d x^{k}\right), \frac{\partial A}{\partial t}\right\rangle f^{2} G_{u_{0}} d V_{g} d t \\
& -r 2 \operatorname{Re} \int_{T_{r}\left(u_{0}\right)}\left\langle x^{i} F_{A, i k} d x^{k},\left[\Lambda_{\omega}, d \omega\right]^{*} F_{A}\right\rangle f^{2} G_{u_{0}} d V_{g} d t \\
& -r 2 \operatorname{Re} \int_{T_{r}\left(u_{0}\right)}\left\langle x^{i} F_{A, i k} d x^{k}, 2 f g^{\alpha \beta} \frac{\partial f}{\partial x^{\alpha}} F_{A, \beta l} d x^{l}\right\rangle f G_{u_{0}} d V_{g} d t \\
& -r 2 \operatorname{Re} \int_{T_{r}\left(u_{0}\right)}\left\langle x^{i} F_{A, i k} d x^{k}, \frac{g^{\alpha \beta} x^{\alpha}}{2\left(t-t_{0}\right)} F_{A, \beta l} d x^{l}\right\rangle f^{2} G_{u_{0}} d V_{g} d t \\
& -r 2 \operatorname{Re} \int_{T_{r}\left(u_{0}\right)}\left\langle x^{i} F_{A}\left(\nabla_{j} \frac{\partial}{\partial x^{i}}, \frac{\partial}{\partial x^{k}}\right) d x^{j} \wedge d x^{k}, F_{A}\right\rangle f^{2} G_{u_{0}} d V_{g} d t \\
& -4 r \int_{T_{r}\left(u_{0}\right)}\left|F_{A}\right|^{2} f^{2} G_{u_{0}} d V_{g} d t,
\end{aligned}
$$

and

$$
\begin{aligned}
I_{2.2}= & r 2 \int_{T_{r}\left(u_{0}\right)}\left(t-t_{0}\right) \frac{\partial}{\partial t}\left|F_{A}\right|^{2} f^{2} G_{u_{0}} d V_{g} d t \\
= & r 4 \operatorname{Re} \int_{T_{r}\left(u_{0}\right)}\left(t-t_{0}\right)\left\langle F_{A}, D_{A} \frac{\partial A}{\partial t}\right\rangle f^{2} G_{u_{0}} d V_{g} d t \\
= & r \operatorname{Re} \int_{T_{r}\left(u_{0}\right)}\left(t-t_{0}\right)\left\langle D_{A}^{*} F_{A}, \frac{\partial A}{\partial t}\right\rangle f^{2} G_{u_{0}} d V_{g} d t \\
& -4 r \operatorname{Re} \int_{T_{r}\left(u_{0}\right)}\left(t-t_{0}\right)\left\langle F_{A}, d\left(f^{2} G_{u_{0}}\right) \wedge \frac{\partial A}{\partial t}\right\rangle d V_{g} d t \\
= & 4 \int_{T_{r}\left(u_{0}\right)}\left(t-t_{0}\right)\left|\frac{\partial A}{\partial t}\right|^{2} f^{2} G_{u_{0}} d V_{g} d t \\
& -r 4 \operatorname{Re} \int_{T_{r}\left(u_{0}\right)}\left(t-t_{0}\right)\left\langle\left[\Lambda_{\omega}, d \omega\right]^{*} F_{A}, \frac{\partial A}{\partial t}\right\rangle f^{2} G_{u_{0}} d V_{g} d t \\
& -r 4 \operatorname{Re} \int_{T_{r}\left(u_{0}\right)}\left(t-t_{0}\right)\left\langle 2 g^{\alpha \beta} \frac{\partial f}{\partial x^{\alpha}} F_{A, \beta l} d x^{l}, \frac{\partial A}{\partial t}\right\rangle f G_{u_{0}} d V_{g} d t \\
& -r 4 \operatorname{Re} \int_{T_{r}\left(u_{0}\right)}\left(t-t_{0}\right)\left\langle\frac{g^{\alpha \beta} x^{\alpha}}{2\left(t-t_{0}\right)} F_{A, \beta l} d x^{l}, \frac{\partial A}{\partial t}\right\rangle f^{2} G_{u_{0}} d V_{g} d t .
\end{aligned}
$$

For simplicity, we set $x \odot F_{A}=\frac{1}{2} x^{i} F_{A, i k} d x^{k}, x \cdot F_{A}=\frac{1}{2} x^{\alpha} g^{\alpha \beta} F_{A, \beta l} d x^{l}$ and $\nabla f \cdot F_{A}=$ $2 g^{\alpha \beta} f^{-1} f_{\alpha} F_{A, \beta l} d x^{l}$, where $f_{\alpha}=\frac{\partial f}{\partial x^{\alpha}}$. Substituting $I_{2.1}$ and $I_{2.2}$ into $I_{2}$, we have 


$$
\begin{aligned}
I_{2}= & 4 r \int_{T_{r}\left(u_{0}\right)} \frac{1}{\left|t-t_{0}\right|}\left|t-t_{0}\right| \frac{\partial A}{\partial t}-\left.x \odot F_{A}\right|^{2} f^{2} G_{u_{0}} d V_{g} d t \\
& +4 r \int_{T_{r}\left(u_{0}\right)} \frac{1}{\left|t-t_{0}\right|}\left\langle x \cdot F_{A}-x \odot F_{A}, x \odot F_{A}-\left|t-t_{0}\right| \frac{\partial A}{\partial t}\right\rangle f^{2} G_{u_{0}} d V_{g} d t \\
& +4 r \int_{T_{r}\left(u_{0}\right)}\left\langle\nabla f \cdot F_{A},\left|t-t_{0}\right| \frac{\partial A}{\partial t}-x \odot F_{A}\right\rangle f^{2} G_{u_{0}} d V_{g} d t \\
& +4 r \int_{T_{r}\left(u_{0}\right)}\left\langle\left[\Lambda_{\omega}, d \omega\right]^{*} F_{A},\left|t-t_{0}\right| \frac{\partial A}{\partial t}-x \cdot F_{A}\right\rangle f^{2} G_{u_{0}} d V_{g} d t \\
& -r 2 \int_{T_{r}\left(u_{0}\right)}\left\langle x^{i} F_{A}\left(\nabla_{j} \frac{\partial}{\partial x^{i}}, \frac{\partial}{\partial x^{k}}\right) d x^{j} \wedge d x^{k}, F_{A}\right\rangle f^{2} G_{u_{0}} d V_{g} d t \\
& -4 r \int_{T_{r}\left(u_{0}\right)}^{\left|F_{A}\right|^{2} f^{2} G_{u_{0}} d V_{g} d t .}
\end{aligned}
$$

By Cauchy inequality, it holds that

$$
\begin{aligned}
I_{2} \geq & r \int_{T_{r}\left(u_{0}\right)} \frac{1}{\left|t-t_{0}\right|}\left|t-t_{0}\right| \frac{\partial A}{\partial t}-\left.x \odot F_{A}\right|^{2} f^{2} G_{u_{0}} d V_{g} d t \\
& -4 r \int_{T_{r}\left(u_{0}\right)} \frac{1}{\left|t-t_{0}\right|}\left|x \cdot F_{A}-x \odot F_{A}\right|^{2} f^{2} G_{u_{0}} d V_{g} d t \\
& -4 r \int_{T_{r}\left(u_{0}\right)}\left|t-t_{0}\right|\left|\nabla f \cdot F_{A}\right|^{2} f^{2} G_{u_{0}} d V_{g} d t \\
& -4 r \int_{T_{r}\left(u_{0}\right)}\left|t-t_{0}\right|\left|\left[\Lambda_{\omega}, d \omega\right]^{*} F_{A}\right|^{2} f^{2} G_{u_{0}} d V_{g} d t \\
& -r 2 \int_{T_{r}\left(u_{0}\right)}\left\langle x^{i} F_{A}\left(\nabla_{j} \frac{\partial}{\partial x^{i}}, \frac{\partial}{\partial x^{k}}\right) d x^{j} \wedge d x^{k}, F_{A}\right\rangle f^{2} G_{u_{0}} d V_{g} d t \\
& -4 r \int_{T_{r}\left(u_{0}\right)}\left|F_{A}\right|^{2} f^{2} G_{u_{0}} d V_{g} d t .
\end{aligned}
$$

By simple calculation, we have

$$
\begin{aligned}
I_{3}= & r \int_{T_{r}\left(u_{0}\right)}\left|F_{A}\right|^{2} x^{i} \frac{\partial\left(f^{2} \sqrt{\operatorname{det}\left(g_{i j}\right)}\right)}{\partial x^{i}} G_{u_{0}} d x d t \\
= & r \int_{T_{r}\left(u_{0}\right)}\left|F_{A}\right|^{2} x^{i} 2 f f_{i} G_{u_{0}} d V_{g} d t \\
& +r \int_{T_{r}\left(u_{0}\right)}\left|F_{A}\right|^{2} x^{i} \frac{\partial \sqrt{\operatorname{det}\left(g_{i j}\right)}}{\partial x^{i}} f^{2} G_{u_{0}} d x d t \\
= & r \int_{T_{r}\left(u_{0}\right)}\left|F_{A}\right|^{2} x^{i} 2 f f_{i} G_{u_{0}} d V_{g} d t \\
& +\frac{r}{2} \int_{T_{r}\left(u_{0}\right)}\left|F_{A}\right|^{2} x^{i} \operatorname{tr}\left(\frac{\partial g}{\partial x^{i}} g^{-1}\right) f^{2} G_{u_{0}} d V_{g} d t .
\end{aligned}
$$


Since $\left|g_{i j}-\delta_{i j}\right| \leq C|x|^{2},\left|\frac{\partial g_{i j}}{\partial x^{k}}\right| \leq C|x|$ and $\left|\Gamma_{j k}^{i}\right| \leq C|x|$, there exists a constant $C_{1}$ such that

$$
\begin{aligned}
& \left|x \cdot F_{A}-x \odot F_{A}\right|^{2}=\left|\frac{1}{2} x^{i}\left(\delta_{j}^{i}-g^{i j}\right) F_{A, j l} d x\right|^{2} \leq C_{1}|x|^{6}\left|F_{A}\right|^{2}, \\
& \left\langle x^{i} F_{A}\left(\nabla_{j} \frac{\partial}{\partial x^{i}}, \frac{\partial}{\partial x^{k}}\right) d x^{j} \wedge d x^{k}, F_{A}\right\rangle \leq C_{1}|x|^{2}\left|F_{A}\right|^{2}, \\
& \operatorname{tr}\left(\frac{\partial g}{\partial x^{i}} g^{-1}\right) \leq C_{1}|x| .
\end{aligned}
$$

In addition, it is easy to check that there exists a constant $C_{2}>0$ such that

$$
\left|\left[\Lambda_{\omega}, d \omega\right]^{*} F_{A}\right|^{2} \leq C_{2}\left|F_{A}\right|^{2} .
$$

Substituting (2.19) and (2.20) into (2.18) and (2.17), we have there exists a constant $C_{3}>0$

$$
\begin{aligned}
\frac{d \Phi(r)}{d r} & \geq r \int_{T_{r}\left(u_{0}\right)} \frac{1}{\left|t-t_{0}\right|}\left|t-t_{0}\right| \frac{\partial A}{\partial t}-\left.x \odot F_{A}\right|^{2} f^{2} G_{u_{0}} d V_{g} d t \\
& -C_{3} r \int_{T_{r}\left(u_{0}\right)} \frac{|x|^{6}}{\left|t-t_{0}\right|}\left|F_{A}\right|^{2} f^{2} G_{u_{0}} d V_{g} d t \\
& -4 r \int_{T_{r}\left(u_{0}\right)}\left|t-t_{0}\right|\left|\nabla f \cdot F_{A}\right|^{2} f^{2} G_{u_{0}} d V_{g} d t \\
& -C_{3} r \int_{T_{r}\left(u_{0}\right)}\left|t-t_{0}\right|\left|F_{A}\right|^{2} f^{2} G_{u_{0}} d V_{g} d t \\
& -C_{3} r \int_{T_{r}\left(u_{0}\right)}|x|^{2}\left|F_{A}\right|^{2} f^{2} G_{u_{0}} d V_{g} d t \\
& -2 r \int_{T_{r}\left(u_{0}\right)}|x||\nabla f||f|\left|F_{A}\right|^{2} G_{u_{0}} d V_{g} d t
\end{aligned}
$$

From [5, p. 99], we have there exists a constant $C_{4}>0$ such that

$$
\begin{aligned}
& r^{-1}\left|t-t_{0} \| x\right|^{6} G_{u_{0}} \leq C_{4}\left(1+G_{u_{0}}\right), \\
& r^{-1}|x|^{2} G_{u_{0}} \leq C_{4}\left(1+G_{u_{0}}\right)
\end{aligned}
$$

holds on $T_{r}\left(u_{0}\right)$. Therefore, we get

$$
\begin{aligned}
& -C_{3} r \int_{T_{r}\left(u_{0}\right)}\left(\frac{|x|^{6}}{\left|t-t_{0}\right|}+\left|t-t_{0}\right|+|x|^{2}\right)\left|F_{A}\right|^{2} f^{2} G_{u_{0}} d V_{g} d t \\
& \geq-C_{5} \Phi(r)-C_{5} r \mathrm{YM}(0),
\end{aligned}
$$

where $C_{5}$ is a positive constant depending on $C_{3}$ and $C_{4}$. At last, we estimate the remaining two terms

$$
-4 r \int_{T_{r}\left(u_{0}\right)}\left|t-t_{0}\right|\left|\nabla f \cdot F_{A}\right|^{2} f^{2} G_{u_{0}} d V_{g} d t
$$

and

$$
-2 r \int_{T_{r}\left(u_{0}\right)}|x|\left|\nabla f\|f\| F_{A}\right|^{2} G_{u_{0}} d V_{g} d t .
$$

Since $\operatorname{supp}(\nabla f) \subseteq B_{R}\left(x_{0}\right) \backslash B_{R / 2}\left(x_{0}\right),|\nabla f| \leq 2 / R$ and $\left|t-t_{0}\right| \leq 4 r^{2}$, we have

$$
\left|t-t_{0}\right|\left|\nabla f \cdot F_{A}\right|^{2} f^{2} G_{u_{0}} \leq \frac{16}{(4 \pi)^{n} R^{2}} r^{2-2 n} \exp \left(-\frac{R^{2}}{64 r^{2}}\right)\left|F_{A}\right|^{2}
$$


and

$$
|x||\nabla f \| f| G_{u_{0}} \leq R \cdot \frac{2}{R}(4 \pi)^{-n} r^{-2 n} \exp \left(-\frac{R^{2}}{64 r^{2}}\right) .
$$

Setting $h(r)=r^{2-2 n} \exp \left(-\frac{R^{2}}{64 r^{2}}\right)$, we have

$$
h^{\prime}(r)=\left(R^{2} / 32-(2 n-2) r^{2}\right) r^{-(2 n+1)} \exp \left(-R^{2} / 64 r^{2}\right) .
$$

So $h(r) \leq\left(\frac{64(n-1)}{e}\right)^{n-1} R^{2-2 n}$. This implies

$$
\begin{aligned}
& -4 r \int_{T_{r}\left(u_{0}\right)}\left|t-t_{0}\right|\left|\nabla f \cdot F_{A}\right|^{2} f^{2} G_{u_{0}} d V_{g} d t \\
\geq & -\frac{C(n) r}{R^{2 n}} \int_{t_{0}-4 r^{2}}^{t_{0}-r^{2}} \int_{B_{R}\left(x_{0}\right)}\left|F_{A}\right|^{2} d V_{g} d t \\
\geq & -\frac{2 C(n) r}{R^{2 n}} \int_{t_{0}-R^{2}}^{t_{0}+R^{2}} \int_{B_{R}\left(x_{0}\right)}\left|F_{A}\right|^{2} d V_{g} d t .
\end{aligned}
$$

Using the same method, we have

$$
-2 r \int_{T_{r}\left(u_{0}\right)}|x||\nabla f||f|\left|F_{A}\right|^{2} G_{u_{0}} d V_{g} d t \geq-\frac{C(n) r}{R^{2 n}} \int_{t_{0}-R^{2}}^{t_{0}+R^{2}} \int_{B_{R}\left(x_{0}\right)}\left|F_{A}\right|^{2} d V_{g} d t .
$$

From the above all, we obtain that there exists a constant $C_{6}>0$ such that

$$
\begin{aligned}
\frac{d \Phi(r)}{d r} & =I_{1}+I_{2}+I_{3} \\
& \geq-C_{6} \Phi(r)-C_{6} r \mathrm{YM}(0)-\frac{C_{6} r}{R^{2 n}} \int_{P_{R}\left(x_{0}, t_{0}\right)}\left|F_{A}\right|^{2} d V_{g} d t .
\end{aligned}
$$

Setting $\operatorname{YM}(0)+R^{-2 n} \int_{P_{R}\left(x_{0}, t_{0}\right)}\left|F_{A}\right|^{2} d V g d t=B$, we have

$$
\frac{d}{d r}\left(e^{C_{6} r} \Phi(r)\right)=e^{C_{6} r}\left(\Phi^{\prime}(r)+C_{6} \Phi(r)\right) \geq-C_{6} r e^{C_{6} r} B
$$

Integrating the two sides along $\left[r_{1}, r_{2}\right]$, we have

$$
\Phi\left(r_{1}\right) \leq \exp \left(C_{7}\left(r_{2}-r_{1}\right)\right) \Phi\left(r_{2}\right)+C_{7}\left(r_{2}^{2}-r_{1}^{2}\right) \mathrm{YM}(0)+C_{7} R^{2-2 n} \int_{P_{R}\left(x_{0}, t_{0}\right)}\left|F_{A}\right|^{2} d V g d t,
$$

where $C_{7}$ is a positive constant depending on the geometry of $X$.

\subsection{Small energy regularity.}

Theorem 2.9. Suppose that $A(t)$ is a smooth solution of the heat flow (1.3), then there exist positive constants $\varepsilon_{0}$ and $\delta_{0}$ such that if for some $0<R<\min \left\{i_{X} / 2, \sqrt{t_{0}} / 2\right\}$, the inequality

$$
R^{2-2 n} \int_{P_{R}\left(u_{0}\right)} e(A)<\varepsilon_{0}
$$

holds, then for any $\delta \in\left(0, \min \left\{\delta_{0}, 1 / 4\right\}\right)$, we have

$$
\sup _{P_{\delta R}\left(x_{0}, t_{0}\right)}\left|F_{A}\right|^{2}<16(\delta R)^{-4}
$$


Proof. For any $\delta \in(0,1 / 4]$, we define the function

$$
f(r)=(2 \delta R-r)^{4} \sup _{P_{r}\left(x_{0}, t_{0}\right)}\left|F_{A}\right|^{2} .
$$

Since $f(r)$ is continuous and $f(2 \delta R)=0$, we have $f(r)$ attains its maximum at a certain point $r_{0} \in[0,2 \delta R)$. We claim that $f\left(r_{0}\right)<16$, this means that for any $r \in[0,2 \delta R)$, we have

$$
(2 \delta R-r)^{4} \sup _{P_{r}\left(x_{0}, t_{0}\right)}\left|F_{A}\right|^{2}<16 .
$$

In particularly, when $r \in[0, \delta R]$, it holds that $\sup _{P_{r}\left(x_{0}, t_{0}\right)}\left|F_{A}\right|^{2}<16 /(2 \delta R-r)^{4}<16(\delta R)^{-4}$.

Assuming that the claim is not true. This means $f\left(r_{0}\right) \geq 16$. Set

$$
\rho_{0}=\left(2 \delta R-r_{0}\right) f\left(r_{0}\right)^{-1 / 4}<\frac{1}{2}\left(2 \delta R-r_{0}\right)=\delta R-r_{0} / 2 .
$$

Rescal the Riemannian metric $\tilde{g}=\rho_{0}^{-2} g$ and $t=t_{1}+\rho^{2} \tilde{t}$, where $\left(x_{1}, t_{1}\right)$ satisfies

$$
\left|F_{A}\right|^{2}\left(x_{1}, t_{1}\right)=\sup _{P_{r_{0}}\left(x_{0}, t_{0}\right)}\left|F_{A}\right|^{2} .
$$

Setting

$$
e_{\rho_{0}}(x, \tilde{t})=\left|F_{A}\right|_{\tilde{g}}^{2}=\rho_{0}^{4}\left|F_{A}\right|_{g}^{2},
$$

we have

$$
e_{\rho_{0}}\left(x_{1}, 0\right)=\rho_{0}^{4}\left|F_{A}\right|^{2}\left(x_{1}, t_{1}\right)=\rho_{0}^{4} \times \frac{f\left(r_{0}\right)}{\left(2 \delta R-r_{0}\right)^{4}}=1
$$

and

$$
\begin{aligned}
\sup _{(x, \tilde{t}) \in \tilde{P}_{1}\left(x_{1}, 0\right)} e_{\rho_{0}}(A)(x, \tilde{t}) & =\rho_{0}^{4} \sup _{P_{\rho_{0}}\left(x_{1}, t_{1}\right)} e(A) \leq \rho_{0}^{4} \sup _{\frac{2 \delta R+r_{0}}{2}\left(x_{1}, t_{1}\right)} e(A) \\
& \leq \rho_{0}^{4}\left(\frac{2 \delta R+r_{0}}{2}\right)^{-4} f\left(r_{0}\right)=16
\end{aligned}
$$

where $\tilde{P}_{1}\left(x_{1}, 0\right)=B_{\rho_{0}}\left(x_{0}\right) \times[-1,1]$. From the Bochner type inequality and (2.21) , on $\tilde{P}_{1}\left(x_{1}, 0\right)$, it holds that

$$
\begin{aligned}
\left(\frac{\partial}{\partial \tilde{t}}-\triangle_{\tilde{g}}\right) e_{\rho_{0}}(x, \tilde{t}) & =\rho_{0}^{6}\left(\frac{\partial}{\partial t}-\triangle_{g}\right) e(A)(x, t) \\
& \leq C \rho_{0}^{6}\left(1+|R i c|_{g}+\left|R_{m}\right|_{g}+\left|F_{A}\right|_{g}\right) e(A) \\
& \leq C^{\prime} e_{\rho_{0}}
\end{aligned}
$$

By the parabolic mean value inequality, we have

$$
1=e_{\rho_{0}}\left(x_{1}, 0\right) \leq C^{\prime \prime} \int_{\tilde{P}\left(x_{1}, 0\right)}\left|F_{A}\right|_{\tilde{g}}^{2} d V_{\tilde{g}} d \tilde{t}
$$

where $C>0$ is a constant depending on the geometry of $X$ and the initial connection $A_{0}$.

Choose normal geodesic coordinates centred at $x_{1}$ and construct cut-off function $\varphi \in C_{0}^{\infty}\left(B_{R / 2}\left(x_{1}\right)\right)$ such that $0 \leq \varphi \leq 1, \varphi \equiv 1$ on $B_{R / 4}\left(x_{1}\right)$ and $|\nabla \varphi| \leq 8 / R$. Taking $r_{1}=\rho$ and $r_{2}=\min \left\{1 / 4, \delta_{0}\right\} R$ 
and applying the monotonicity formula, we have

$$
\begin{aligned}
\int_{\tilde{P}_{1}\left(x_{1}, 0\right)}\left|F_{A}\right|_{\tilde{g}}^{2} d V_{\tilde{g}} & d \tilde{t}=\rho_{0}^{2-2 n} \int_{P_{\rho_{0}}\left(x_{1}, t_{1}\right)}\left|F_{A}\right|_{g}^{2} d V_{g} d t \\
\leq & C \rho_{0}^{2} \int_{P_{\rho_{0}}\left(x_{1}, t_{1}\right)}\left|F_{A}\right|_{g}^{2} G_{\left(x_{1}, t_{1}+2 \rho_{0}^{2}\right)} \varphi^{2} d V_{g} d t \\
\leq & C \rho_{0}^{2} \int_{T_{\rho_{0}}\left(x_{1}, t_{1}+2 \rho_{0}^{2}\right)}\left|F_{A}\right|_{g}^{2} G_{\left(x_{1}, t_{1}+2 \rho_{0}^{2}\right)} \varphi^{2} d V_{g} d t \\
\leq & C e^{C\left(r_{2}-\rho_{0}\right)} r_{2}^{2} \int_{T_{r_{2}}\left(x_{1}, t_{1}+2 \rho_{0}^{2}\right)}\left|F_{A}\right|_{g}^{2} G_{\left(x_{1}, t_{1}+2 \rho_{0}^{2}\right)} \varphi^{2} d V_{g} d t \\
& +C\left(r_{2}^{2}-\rho_{0}^{2}\right) Y M(0)+C(R / 2)^{2-2 n} \int_{P_{R / 2}\left(x_{1}, t_{1}\right)}\left|F_{A}\right|^{2} d V_{g} d t \\
\leq & C \delta_{0}^{2-2 n} R^{2-2 n} \int_{P_{R}\left(x_{0}, t_{0}\right)}\left|F_{A}\right|^{2} d V_{g} d t+C \delta_{0}^{2} R^{2} Y M(0) \\
& +C \delta_{0}^{2-2 n} R^{2-2 n} \int_{P_{R}\left(x_{0}, t_{0}\right)}\left|F_{A}\right|^{2} d V_{g} d t \\
\leq & \tilde{C}\left(\delta_{0}^{2-2 n} \varepsilon_{0}+\delta_{0}^{2} R^{2} Y M(0)\right),
\end{aligned}
$$

where the constants depend on the geometry of $X$ and the initial data $A_{0}$. From (2.22) and (2.23), we have $1 \leq C^{\prime \prime} \tilde{C}\left(\delta_{0}^{2-2 n} \varepsilon_{0}+\delta_{0}^{2} R^{2} Y M(0)\right)$. Thus, choosing $\delta_{0}$ and $\varepsilon_{0}$ properly, we can obtain a contradiction .

\section{Proof of Theorem 1.1}

Using the same argument as that in the proof of Theorem 2 in Bando and Siu's paper ([2]), we have

Theorem 3.1. Let $X$ be an $n$-dimensional complex manifold, $g$ a Hermitian metric on $X$ with associated $(1,1)$-form $\omega$. Let $(E, h)$ be a holomorphic vector bundle with a Hermitian metric $h$ over $X \backslash S$, where $S$ is a closed subset with locally finite Hausdorff measure of real co-dimension 4.If the curvature $F_{h}$ is locally integrable, then

(1) $E$ can be extended to the whole $X$ as a reflexive sheaf $\mathcal{E}$, and for any local section $s \in \Gamma(U, \mathcal{E})$, $\log ^{+} h(s, s)$ belongs to $H_{l o c}^{1}$;

(2) If $\Lambda_{\omega} F$ is locally bounded, then $h$ is locally bounded and $h \in W_{\text {loc }}^{2, p}$ for any finite $p$ where $\mathcal{E}$ is locally free;

(3) If $(E, h)$ is Hermitian-Einstein, then $h$ smoothly extends as a Hermitian-Einstein metric over the place where $\mathcal{E}$ is locally free.

\section{Proof of Theorem 1.1}

(1) Step 1 Construct the closed set $\Sigma$ of Hausdorff codimension at least 4

From Lemma 2.3, we have for arbitrary sequence $t_{k} \rightarrow \infty$ and $a>0$, it holds that

$$
\int_{t_{k}-a}^{t_{k}+a} \int_{X}\left|\frac{\partial A}{\partial t}\right|^{2} d V_{g} d t \rightarrow 0, \quad t_{k} \rightarrow \infty .
$$

Then for arbitrary $\epsilon>0$, there exists $K \in \mathbb{Z}^{+}$, such that when $k \geq K$, it holds that

$$
\int_{t_{k}-a}^{t_{k}+a} \int_{X}\left|\frac{\partial A}{\partial t}\right|^{2} d V_{g} d t<\epsilon
$$


Construct the set

$$
\Sigma=\bigcap_{0<r<i_{X}}\left\{x \in X, \lim _{k \rightarrow \infty} \inf r^{4-2 n} \int_{B_{r}(x)} e(A)\left(\cdot, t_{k}\right) d V_{g} \geq \varepsilon_{1}\right\}
$$

where $\varepsilon_{1}$ is determined below.

For $x_{1} \in X \backslash \Sigma$, there exists $r_{1}>0$, such that when $t_{k}$ is sufficiently large, we have

$$
r_{1}^{4-2 n} \int_{B_{r_{1}}\left(x_{1}\right)} e(A)\left(\cdot, t_{k}\right) d V_{g}<\varepsilon_{1} .
$$

Set $s=t_{k}-r_{1}^{2}, \tau=t_{k}+r_{1}^{2}$. Applying Lemma 2.4, for any $t \in[s, \tau]$, we have

$$
\begin{aligned}
\int_{B_{r_{1} / 2}\left(x_{1}\right)} e(A)(\cdot, t) d V_{g} & \leq \int_{B_{r_{1}}\left(x_{1}\right)} e(A)\left(\cdot, t_{k}\right)+2 \int_{t_{k}-r_{1}^{2}}^{t_{k}+r_{1}^{2}} \int_{X}\left|\frac{\partial A}{\partial t}\right|^{2} d V_{g} d t \\
& +C\left(\operatorname{YM}(0) \int_{t_{k}-r_{1}^{2}}^{t_{k}+r_{1}^{2}} \int_{X}\left|\frac{\partial A}{\partial t}\right|^{2} d V_{g} d t\right)^{1 / 2} \\
& +C i_{X}\left(\operatorname{YM}(0) \int_{t_{k}-r_{1}^{2}}^{t_{k}+r_{1}^{2}} \int_{X}\left|\frac{\partial A}{\partial t}\right|^{2} d V_{g} d t\right)^{1 / 2} \\
& \leq \int_{B_{r_{1}}\left(x_{1}\right)} e(A)\left(\cdot, t_{k}\right)+2 \epsilon+C\left(1+i_{X}\right)(\operatorname{YM}(0) \epsilon)^{1 / 2}
\end{aligned}
$$

Consider

$$
r_{1}^{2-2 n} \int_{P_{r_{1} / 2}\left(x_{1}, t_{k}\right)} e(A)(\cdot, t) d V_{g} d t=r_{1}^{2-2 n} \int_{t_{k}-\left(r_{1} / 2\right)^{2}}^{t_{k}+\left(r_{1} / 2\right)^{2}} \int_{B_{r_{1} / 2}\left(x_{1}\right)} e(A)(\cdot, t) d V_{g} d t
$$

Substituting (3.1) into (3.2), we have

$$
\begin{aligned}
& r_{1}^{2-2 n} \int_{t_{k}-\left(r_{1} / 2\right)^{2}}^{t_{k}+\left(r_{1} / 2\right)^{2}} \int_{B_{r_{1} / 2}\left(x_{1}\right)} e(A)(\cdot, t) d V_{g} d t \\
\leq & r_{1}^{2-2 n} \int_{t_{k}-\left(r_{1} / 2\right)^{2}}^{t_{k}+\left(r_{1} / 2\right)^{2}} \int_{B_{r_{1}}\left(x_{1}\right)} e(A)\left(\cdot, t_{k}\right) d V_{g} d t \\
& +C r_{1}^{4-2 n}\left(\epsilon+\left(1+i_{X}\right)(Y M(0) \epsilon)^{1 / 2}\right) \\
= & \frac{1}{2} r_{1}^{4-2 n} \int_{B_{r_{1}\left(x_{1}\right)}} e(A)\left(\cdot, t_{k}\right) d V_{g}+C r_{1}^{4-2 n}\left(\epsilon+\left(1+i_{X}\right)(Y M(0) \epsilon)^{1 / 2}\right)
\end{aligned}
$$

Choosing $\varepsilon_{1}=\varepsilon_{0} / 4^{n-1}$ and $\epsilon$ such that $C 2^{2 n-2} r_{1}^{4-2 n}\left(\epsilon+\left(1+i_{X}\right)(Y M(0) \epsilon)^{1 / 2}\right) \leq \varepsilon_{0} / 2$, we have

$$
\left(\frac{r_{1}}{2}\right)^{2-2 n} \int_{P_{r_{1} / 2}\left(x_{1}, t_{k}\right)} e(A)(\cdot, t) d V_{g} d t \leq \varepsilon_{0} .
$$

where $\varepsilon_{0}$ is the constant in Theorem 2.9, Applying the small energy regularity theorem, we have

$$
\sup _{P_{\delta_{0} r_{1}}\left(x_{1}, t_{k}\right)} e(A)(\cdot, \cdot) \leq C\left(\delta_{0} r_{1}\right)^{-4}
$$

where $\delta_{0}$ is the constant in Theorem 2.9. 
It is easy to check that for any $x \in B_{\delta_{0} r_{1}}\left(x_{1}\right)$, we can choose small enough $r_{x}$ such that $B_{r_{x}}(x) \subseteq B_{\delta_{0} r_{1}}\left(x_{1}\right)$ and

$$
r_{x}^{4-2 n} \int_{B_{r_{x}}(x)} e(A)\left(\cdot, t_{k}\right) \leq r_{x}^{4-2 n} r_{x}^{2 n} C\left(\delta_{0} r_{1}\right)^{-4}=C\left(\frac{r_{x}}{\delta_{0} r_{1}}\right)^{4}<\varepsilon_{1},
$$

that is $B_{\delta_{0} r_{1}}\left(x_{1}\right) \subseteq X \backslash \Sigma$ and $\Sigma$ is closed.

In fact $\mathcal{H}^{2 n-4}(\Sigma)<\infty$. Since $\Sigma$ is closed, we have for any $\delta>0$, there exist finite geodesic balls $\left\{B_{r_{i}}\left(x_{i}\right)\right\}_{i \in \Gamma}$, where $x_{i} \in \Sigma, r_{i}<\delta$, such that

- $\Sigma \subseteq \cup_{i \in \Gamma} B_{r_{i}}$,

- when $i \neq j, B_{r_{i} / 2}\left(x_{i}\right) \cap B_{r_{j} / 2}\left(x_{j}\right)=\emptyset$.

Since $x_{i} \in \Sigma$, we have

$$
r_{i}^{4-2 n} \int_{B_{r_{i} / 2}} e(A)\left(\cdot, t_{k}\right) d V_{g}>2^{2 n-4} \varepsilon_{1},
$$

for $t_{k}$ is sufficiently large. This implies

$$
r_{i}^{2 n-4}<\varepsilon_{1}^{-1} 2^{4-2 n} \int_{B_{r_{i} / 2}} e(A)\left(\cdot, t_{k}\right) d V_{g} .
$$

Thus we have

$$
\sum_{i \in \Gamma} r_{i}^{2 n-4} \leq \varepsilon_{1}^{-1} 2^{4-2 n} \int_{\cup B_{r_{i} / 2}} e(A)\left(\cdot, t_{k}\right)<\varepsilon_{1}^{-1} 2^{4-2 n} \mathrm{YM}(0)<+\infty .
$$

This implies that $\mathcal{H}^{2 n-4}(\Sigma) \leq \varepsilon_{1}^{-1} 2^{4-2 n} \mathrm{YM}(0)<\infty$.

Step 2 The convergence in $X \backslash \Sigma$

From the above, we have that for any $x_{0} \in X \backslash \Sigma$, there exists $r_{0}$, when $t_{k}$ is sufficiently large, it holds that

$$
\sup _{P_{r_{0}}\left(x_{0}, t_{k}\right)} e(A)(\cdot, \cdot) \leq C .
$$

Applying the Uhlenbeck weak compactness theorem ([21] ), there exists subsequence $\left\{t_{k^{\prime}}\right\} \subseteq\left\{t_{k}\right\}$ and gauge transformations $\sigma\left(k^{\prime}\right)$ such that $\sigma\left(k^{\prime}\right)\left(A\left(t_{k^{\prime}}\right)\right)$ converges to the connection $A_{\infty}$ of limiting bundle $\left(E_{\infty}, H_{\infty}\right)$ in weak $W_{l o c}^{1,2}(X \backslash \Sigma)$ sense and $A_{\infty}$ satisfies

$$
D_{A_{\infty}} \Lambda_{\omega} F_{A_{\infty}}=0 \text {. }
$$

In fact, over $X \backslash \Sigma$, it holds that $\sigma\left(k^{\prime}\right)\left(A\left(t_{k}\right)\right)$ converges to $A_{\infty}$ in $\mathcal{C}_{\text {loc }}^{\infty}$ sense. For any $x_{0} \in X \backslash \Sigma$, there exists small enough $r_{0}$, such that when $t_{k}$ is sufficiently large, we have

$$
\sup _{P_{r_{0}}\left(x_{0}, t_{k}\right)}\left|F_{A}\right|^{2} \leq C
$$

Therefore, over $P_{r_{0}}\left(x_{0}, t_{k}\right)$, we have

$$
\begin{aligned}
\left(\triangle-\frac{\partial}{\partial t}\right)\left|F_{A}\right|^{2} & \geq 2\left|\nabla_{A} F_{A}\right|^{2}-C\left(1+|R i c|+|R m|+\left|F_{A}\right|\right)\left|F_{A}\right|^{2}-C\left|F_{A}\right|\left|\nabla_{A} F_{A}\right| \\
& \geq\left|\nabla_{A} F_{A}\right|^{2}-C\left|F_{A}\right|^{2} .
\end{aligned}
$$

Assume that there exist $r_{j}, \quad j=0, \cdots, l-1$ such that

$$
\sup _{P_{r_{j}}\left(x_{0}, t_{k}\right)}\left|\nabla_{A}^{j} F_{A}\right|^{2} \leq C
$$


By a similar proof as that in Lemma 2.7, we have there exists $r_{l}$ such that

$$
\begin{aligned}
\left(\triangle-\frac{\partial}{\partial t}\right)\left|\nabla_{A}^{l} F_{A}\right|^{2} & \geq 2\left|\nabla_{A}^{l+1} F_{A}\right|^{2}-C\left|\nabla_{A}^{l} F_{A}\right|\left|\nabla_{A}^{l+1} F_{A}\right|-C\left|\nabla_{A}^{l} F_{A}\right|^{2}-C\left|\nabla_{A}^{l} F_{A}\right| \\
& \geq\left|\nabla_{A}^{l+1} F_{A}\right|^{2}-C\left|\nabla_{A}^{l} F_{A}\right|^{2}-C .
\end{aligned}
$$

in $P_{r_{l}}\left(x_{0}, t_{k}\right)$. This implies for any $j=1, \cdots, l$, we have

$$
\int_{P_{r_{j}}\left(x_{0}, t_{k}\right)}\left|\nabla_{A}^{j} F_{A}\right|^{2} \leq C .
$$

From the parabolic mean value inequality, there exists $\delta>0$ such that

$$
\sup _{P_{\delta r_{l}}\left(x_{0}, t_{k}\right)}\left|\nabla_{A}^{l} F_{A}\right|^{2} \leq C .
$$

Using Donaldson's diagonal technique in [11, Theorem 4.4.8], there exists subsequence $\left\{t_{k_{i}}\right\}$ and smooth gauge transformation $\left\{\sigma_{k_{i}}\right\}$ such that $\sigma_{k_{i}}\left(A\left(t_{k_{i}}\right)\right)$ converges to $A_{\infty}$ in $\mathcal{C}_{\text {loc }}^{\infty}(X \backslash \Sigma)$ sense and $A_{\infty}$ satisfies equation (1.4).

(2) Since $\sqrt{-1} \Lambda_{\omega} F_{A_{\infty}}$ is parallel and $\left(\sqrt{-1} \Lambda_{\omega} F_{A_{\infty}}\right)^{* H_{\infty}}=\sqrt{-1} \Lambda_{\omega} F_{A_{\infty}}$, we can decompose $\left(E_{\infty}, H_{\infty}\right)$ according to the eigenvalues of $\sqrt{-1} \Lambda_{\omega} F_{\infty}$ over $X \backslash \Sigma$

$$
E_{\infty}=\oplus_{i=1}^{l} E_{\infty}^{i}
$$

Setting $H_{\infty}^{i}=\left.H_{\infty}\right|_{E_{\infty}^{i}}, A_{\infty}^{i}=\left.A_{\infty}\right|_{E^{i}}$, we have $A_{\infty}^{i}$ is a Hermitian-Einstein connection on $\left(E_{\infty}^{i}, H_{\infty}^{i}\right)$, i.e.

$$
\sqrt{-1} \Lambda_{\omega} F_{A_{\infty}^{i}}=\lambda_{i} \operatorname{Id}_{E_{\infty}^{i}}
$$

From Lemma 2.3, we have $\mathrm{YM}(\mathrm{t})$ is decreasing along the flow. So it holds that

$$
\int_{X \backslash \Sigma}\left|F_{A_{\infty}}\right|_{H_{\infty}}^{2}<\infty .
$$

In addition that $\mathcal{H}^{2 n-4}(\Sigma)<\infty$ and $H_{\infty}^{i}$ satisfies the Hermitian-Einstein equation, we have that every $\left(E_{\infty}^{i}, \bar{\partial}_{A_{\infty}^{i}}\right)$ can be extended to the whole $X$ as a reflexive sheaf (also denoted by $\left.\left(E_{\infty}^{i}, \bar{\partial}_{A_{\infty}^{i}}\right)\right)$ and $H_{\infty}^{i}$ can be smoothly extended over the place where the sheaf $\left(E_{\infty}^{i}, \bar{\partial}_{A_{\infty}^{i}}\right)$ is locally free by Theorem 3.1 .

\section{REFERENCES}

[1] M.Atiyah and R.Bott, The Yang-Mills equations ovet Riemann surfaces, Philos. Trans. Roy. Soc. London Ser. A 308 , no. 1505, pp. 523-615,1983.

[2] S.Bando and Y.T.Siu, Stable sheaves and Einstein-Hermitian metrics. In: Geometry and analysis on complex manifolds, World Scientific Publishing, River Ed

[3] Y.M, Chen and C.L. Shen, Monotonicity formula and small action regularity for Yang-Mills flows in higher dimensions. Calc. Var. Partial Differential Equations. 2(4), pp. 389-403, 1994.

[4] Y.M, Chen and C.L. Shen, Evolution problem of Yang-Mills flow over 4-dimensional manifold. Variational methods in nonlinear analysis (Erice, 1992), pp. 63-66, Gordon and Breach, Basel, 1995.

[5] Y.M. Chen and M. Struwe, Existence and partial regularity results for the heat flow for harmonic maps. Math. Z. 201(1), pp. 83-103, 1989.

[6] G.Daaskalopoulos, The topology of the spaces of stable bundles on Riemann surface, J. Differential Geom. 36, no. 3, pp. 699-746, 1992. 
[7] G. Daskalopoulos and R. Wentworth, Convergence properties of the Yang-Mills flow on Kähler surfaces, J. Reine Angew. Math. 575, pp.69-99, 2004.

[8] J.P. Demailly, Complex analytic and differential geometry. book online https://www-fourier.ujf-grenoble.fr/ demailly/books.html

[9] S.K.Donaldson, Anti self-dual Yang-Mills connections over complex algebraic surfaces and stable vector bundles. Proc. London Math. Soc. 50(1), pp. 1-26, 1985.

[10] S.K. Donaldson, Infinite determinants, stable bundles and curvature. Duke Math. J. 54(1), pp. 231-247, 1987.

[11] S.K. Donaldson and P.B. Kronheimer, The geometry of four-manifolds. Clarendon Press, Oxford,1990.

[12] P. Gauduchon, La 1-forme de torsion d'une variété hermitienne compacte. Math.Ann. 267(4), pp. 495-518, 1984.

[13] M.C. Hong and G. Tian, Asymptotical behaviour of the Yang-Mills flow and singular Yang-Mills connections, Math.Ann. 330(3),pp. 441-472, 2004.

[14] J. Li and S.T. Yau, Hermitian-Yang-Mills connection on non-Kähler manifolds. In: Mathematical aspects of string theory, World Scientific, pp. 560-573, 1987.

[15] J.Jost and S.T.Yau, A nonlinear elliptic system for maps from Hermitian to Riemannian manifolds and rigidity theorems in Hermitian geometry. Acta Math. 170(2),pp. 221-254, 1993.

[16] J.Y. Li and X. Zhang, The limit of the Yang-Mills-Higgs flow on Higgs bundles, Int. Math. Res. Not. 1, pp.232-276, 2017.

[17] J.Li, C.J.Zhang and X.Zhang, The limit of the Hermitian-Yang-Mills flow on reflexive sheaves, Adv. Math. 325, pp.165-214, 2018.

[18] M. Lübke and A. Teleman, The Kobayashi-Hitchin correspondence. World Scientific Publishing, 1995.

[19] J. McNamara and Y. Zhao, Limiting behavior of Donaldson's heat flow on non-Kähler surfaces. arXiv:1403.8037.

[20] B. Sibley, Asymptotics of the Yang-Mills flow for holomorphic vector bundles over K?hler manifolds: the canonical structure of the limit., J. Reine Angew. Math. 706, 123-191,2015.

[21] K.K. Uhlenbeck, Connections with $L^{p}$ bounds on curvarure. Comm. Math. Phys. 83(1), pp.31-42,1982.

[22] K.K. Uhlenbeck, A priori estimates for Yang-Mills fields, unpublished manuscript.

[23] K.K. Uhlenbeck and S.T. Yau, On the existence of Hermitian-Yang-Mills connections in stable vector bundles. Comm. Pure Appl. Math. 39S, pp. S257-S293, 1986.

[24] X. Zhang, Hermitian-Einstein metrics on holomorphic vector bundles over Hermitian manifolds. J. Geom. Phys. 53(3), pp. 315-335, 2005.

Yanci Nie, School of Mathematical Sciences, Xiamen University, Xiamen, 361005,

E-mail address: nieyanci@xmu.edu.cn

Xi Zhang, Key Laboratory of Wu Wen-Tsun Mathematics, Chinese Academy of Sciences, School of Mathematical Sciences, University of Science and Technology of China, Hefei, 230026,P.R. CHINA,

E-mail address: mathzx@ustc.edu.cn 